\title{
Yod
}

Revue des études hébraïques et juives

19 | 2014

Aharon Appelfeld, cinquante ans d'écriture

\section{Aharon Appelfeld et Philip Roth : le réel, l'imaginaire, le double et le fantastique}

Aharon Appelfeld and Philip Roth: on History and Fiction, the "Doppelganger" and the Fantastic Genre

אהרון אפלפלד ופיליפ רות: על היסטוריה ובדיון, הכפיל וסיפורת הפנטסיה

\section{Orly Toren}

\section{(2) OpenEdition}

\section{Journals}

Édition électronique

URL : https://journals.openedition.org/yod/2028

DOI : $10.4000 /$ yod. 2028

ISSN : 2261-0200

Éditeur

INALCO

\section{Édition imprimée}

Date de publication : 30 mai 2014

ISBN : 978-2-85837-214-6

ISSN : 0338-9316

\section{Référence électronique}

Orly Toren, « Aharon Appelfeld et Philip Roth : le réel, l'imaginaire, le double et le fantastique », Yod [En ligne], 19 | 2014, mis en ligne le 16 avril 2014, consulté le 08 juillet 2021. URL : http:// journals.openedition.org/yod/2028; DOI : https://doi.org/10.4000/yod.2028

Ce document a été généré automatiquement le 8 juillet 2021.

\section{(ब) $(1) \Theta$}

Yod est mis à disposition selon les termes de la Licence Creative Commons Attribution - Pas d'Utilisation Commerciale 4.0 International. 


\title{
Aharon Appelfeld et Philip Roth : le réel, l'imaginaire, le double et le fantastique
}

\author{
Aharon Appelfeld and Philip Roth: on History and Fiction, the "Doppelganger" \\ and the Fantastic Genre \\ אהרון אפלפלד ופיליפ רות: על היסטוריה ובדיון, הכפיל וסיפורת הפנטסיה
}

\section{Orly Toren}

\section{Introduction}

1 À l'inverse du nombre important d'ouvrages de recherche consacrés aux camps d'extermination nazis, la déportation en Transnistrie n'était connue pendant des dizaines d'années que par des récits oraux de survivants et quelques témoignages écrits, publiés le plus souvent à compte d'auteur. Ce n'est qu'à partir de la fin des années 1990 qu'un nombre croissant de travaux de recherche a jeté un nouvel éclairage sur la déportation des Juifs de Roumanie. Aussi, pendant longtemps, les rescapés de Transnistrie sont-ils restés, dans un certain sens, les «marginaux » de la Shoah, ceux dont on ne disait rien pendant les commémorations, ceux dont on ne savait rien, ceux dont la souffrance comptait moins dans la "hiérarchie» des victimes. Ce sont précisément ces « laissés pour compte » de la Shoah et leurs enfants, dont je fais partie, ceux de ladite "deuxième génération", qui, intellectuels ou non, ont trouvé en Appelfeld le seul auteur à donner une légitimité et une dignité littéraire à leur vécu.

2 J'ai grandi en écoutant ces mots mystérieux qui revenaient dans les conversations des adultes : Transnistrie, Dniestr, Bug, Moghilev, Berschad, Djurin, Lager, la faim, le froid, le typhus, les trains de marchandises, les marches forcées. Mais en deçà des détails, aussi minutieux fussent-ils, il demeurait dans ces témoignages quelque chose d'indéchiffrable, une douleur intense qui ne se disait pas, qui était transmise sans mots et à laquelle Appelfeld, contrairement à mon entourage, a su donner corps et forme à 
travers ses romans. Certes, ses mots étaient voilés, frustrants par leur implicite, mais son art de conteur restituait la cohérence des témoignages fragmentaires.

\section{Le Temps des prodiges : la page blanche et l'« indicible»}

3 J'ai rencontré Appelfeld pour la première fois en 1988 lorsque, journaliste et critique littéraire en Israël, je l'ai interviewé pour l'hebdomadaire Yedioth Yerushalayim ${ }^{1}$ à l'occasion de la sortie de son roman Katerina ${ }^{2}$ aux éditions Keter. "Comment savez-vous où se trouve la Bucovine ? Où avez-vous entendu parler de la Transnistrie? » m'avait-il demandé, étonné. Je ne savais pas encore que trois ans après notre conversation j'irais moi-même en Transnistrie en compagnie de ma mère. Ce n'est qu'au retour de ce voyage que j'ai lu, pour la première fois, Le Temps des prodiges ${ }^{3}$. Si je pensais avoir «compris» en visitant les lieux la part occulte de cette expérience de la déportation qui n'était pas la mienne, mais qui l'était devenue indirectement, comme "par procuration ", j'ai saisi, à travers la lecture de ce roman que le " mystère » qui m'avait hanté tout au long de mon enfance et de ma jeunesse était contenu dans la page blanche qui sépare les deux récits qui forment Le Temps des prodiges. C'est là, dans le non-dit, que résidait l'abîme qui séparait la vie de l'enfant déporté à la fin de la première partie de celle de l'adulte, Bruno, qui retourne dans sa ville natale quelques années après la guerre. L'art du conteur qu'est Appelfeld est celui du silence.

Ainsi, dans sa préface à l'édition française du Temps des prodiges, Marianne Véron oppose la perception du narrateur à celle du lecteur :

Appelfeld impose l'évocation de ce que nous savons par une extraordinaire sobriété du récit. [...] c'est uniquement le quotidien et ses infimes lézardes qu'il nous présente - agrandies par l'ombre projetée par la suite, que nous, nous savons.

Si le jeune narrateur, lui, ne sait pas où se dirige le train de marchandises, dont l'ébranlement clôture la première partie du roman, «nous, nous restons pétrifiés devant l'abîme que cache cet euphémisme $»^{4}$.

Peut-on traduire le non-dit en mots? Appelfeld s'y est toujours refusé. Cette page blanche illustre à elle seule ce que Alexis Nouss définit comme le "récit de l'indicible ", catégorie dans laquelle il classe l'œuvre des écrivains traitant de l'expérience génocidaire et concentrationnaire. Notion empruntée à Wittgenstein, "l'indicible " ("Das Unausprechbare») défait la logique qui découle, d'une part, de la notion du réel comme référent et, d'autre part, de la notion du langage comme médiation :

L'indicible mine conceptuellement l'idée de représentation: l'indicible n'est pas représentable [...] il suggère un réel non référentiel, un réel qui, pour n'en être pas moins réel, ne se dit pas ${ }^{5}$.

7 Si donc l'«indicible", ce qui ne peut pas être dit, est le noyau de l'art narratif d'Appelfeld et que l'indicible relate une forme de « réel non-référentiel », ne renvoie-til pas aux confins entre le réel et l'irréel ? Le rêve et la réalité ? Enfin, au fantastique ?

8 Aussi, le non-dit, l'indicible, est-il étroitement lié au mystère et c'est précisément le mystère du surnaturel que sollicite le titre du roman Le Temps des prodiges, que l'on pourrait également traduire par L'ère des merveilles, dont les "prodiges", voire les «merveilles », ne sont explicités nulle part. De quels «prodiges» s'agit-il ? De quelles «merveilles»? 
9 Un prodige, nous dit le dictionnaire, est un " événement extraordinaire de caractère magique » ou encore, «Acte, action extraordinaire ${ }^{6}$. Or, c'est précisément ce mot, au pluriel, פלאות, «prodiges » et son synonyme " merveilles » qui renvoient à la fois aux miracles, à la fin heureuse des temps qui est celle de l'avènement du Messie tout comme aux contes folkloriques dits merveilleux où prolifèrent, on le sait, des êtres maléfiques et des événements terrifiants dont les protagonistes ne survivent que par les conventions du genre. Or, si Appelfeld impose, par le hors-texte et le paratexte du titre cette association d'idées pour le moins paradoxale entre le réalisme de la littérature du désastre et le fantastique qui remet en question la perception du réel, il oppose délibérément la convention du happy end du " merveilleux » et du « miracle » aux récits de vie des protagonistes de ce roman empreint de nostalgie, de douleur, d'amertume et de résignation dont chacune des deux parties se termine par le triomphe du Mal. Pourtant, c'est précisément ce paradoxe qui permet de mettre en relation les sombres «prodiges » auquel la page blanche qui sépare les deux parties du roman fait allusion avec le genre fantastique dont le conte merveilleux fait partie et la notion de « l'indicible » qui traduit ce qui est au-delà de l'entendement humain.

Comme la poésie de Paul Celan, son aîné de 12 ans, originaire lui aussi de Czernowitz et déporté lui aussi en Transnistrie, la prose d'Appelfeld suggère l'horreur par un style résolument minimaliste. L'économie du spectaculaire, l'ascèse du verbe le rapprochent dans la fiction de ce que Primo Levi a défini, pour le récit factuel, comme «l'étude dépassionnée de certains aspects de l'âme humaine $»^{7}$.

11 Le Temps des prodiges en est l'illustration. Dans la première partie, le narrateur homodiégétique, un garçon de 13 ans, juif autrichien, fils d'un écrivain réputé disciple de Kafka et ami de Stefan Zweig, raconte à travers les détails du quotidien la déchéance de la Bucovine juive. Féru de culture germanique, imbu de ce qu'Appelfeld appelle «l'auto-antisémitisme", ce microcosme juif très particulier, produit de l'extension territoriale de l'Empire austro-hongrois, a été détruit, suggère-t-il, par la haine de soi tout autant que par les nazis. Cible d'une campagne de calomnies dans la presse, le père du narrateur est accusé d'être un " parasite s'abreuvant du sang de la sainte Autriche » et son œuvre dénoncée comme " putride et malfaisante ». On est en 1938 ; les critiques, naguère élogieux à l'égard de cet écrivain, retournent leur veste. Conspué par ses anciens admirateurs, cet intellectuel assimilé découvre le "défaut inné » qu'est son origine juive et consacre désormais toute son énergie à extirper de son œuvre et de son esprit tout ce qui pourrait s'y trouver encore de juif. Son inspiration tarie, il cesse d'écrire. Ce « défaut » inné que le père du narrateur décèle en lui-même, sa judaïté, est le pan moral du handicap physique qui hante l'œuvre d'Appelfeld. C'est le drame des écrivains juifs germanophones sous Hitler illustré par les propos prémonitoires de Kafka en 1921 :

Ils vivaient entre trois impossibilités [...]; l'impossibilité de ne pas écrire, l'impossibilité d'écrire en allemand, l'impossibilité d'écrire autrement, à quoi on pourrait ajouter une quatrième impossibilité, l'impossibilité d'écrire [...], c'était donc une littérature impossible de tous côtés, une littérature de Tziganes qui avaient volé l'enfant allemand au berceau ${ }^{8}$.

Le narrateur, jeune adolescent qui observe ces signes précurseurs sans pouvoir les conceptualiser, est pris d'une angoisse qui va croissant. La première partie du Temps des prodiges, qui porte le même titre et se présente comme un roman bref à part entière, s'achève sur ces mots : "Le lendemain, nous étions déjà enchaînés dans un train de marchandises qui faisait route vers le sud.» Le «sud», ici, est un euphémisme par 
lequel Appelfeld désigne les camps de Transnistrie où il a été déporté, région située en effet au sud-est de Czernowitz, sa ville natale. Comme dans la ballade de Goethe, Le roi des Aulnes', que le jeune Appelfeld, élève au Gymnasium allemand ne pouvait méconnaître, le père du jeune narrateur, obtus et aveugle, n'a pas su déchiffrer les signes et n'a pas su sauver son fils de l'Elfe maléfique.

La deuxième partie du roman intitulée Quand tout fut accompli des années plus tard et qui, comme la première, se présente comme un récit indépendant et dont la voix est celle d'un narrateur hétérodiégétique à la troisième personne, raconte l'histoire de Bruno, un adulte qui revient dans sa ville natale en Autriche vers 1965. S'agit-il d'une bourgade de l'Autriche d'après-guerre? D'une ville de la Bucovine roumaine ou ukrainienne? Appelfeld n'en dit pas plus qu'il ne dévoile si Bruno est le jeune narrateur de la première partie devenu adulte. Le prénom de l'enfant-narrateur n'y avait d'ailleurs jamais été mentionné. Seuls les personnages qu'il retrouve dans cette ville sans nom créent le lien entre les deux parties: Louise, la bonne plantureuse, objet de ses premiers fantasmes sexuels (et dont on retrouvera les avatars dans les personnages appelfeldiens de Katerina ou Mariana), est désormais une vieille femme décrépite. Brum, le juif converti et métamorphosé en authentique éleveur de bétail autrichien refuse obstinément de reconnaître en ce Bruno adulte l'enfant qu'il avait pourtant fréquenté dans le passé et la bande des "métis ", enfants naturels de bourgeois juifs et de paysannes ruthènes, sont devenus des voyous. L'enfant candide a laissé la place à l'adulte désabusé. Ce qui s'est passé pendant les vingt-cinq ans qui séparent le récit de l'enfant de celui de l'adulte reste inexprimé. La première et la deuxième partie, qui se présentent comme deux nouvelles distinctes, qui portent, chacune, son propre titre, sont séparées par une page blanche. C'est cette page blanche qui renferme l'« indicible », lieu des "prodiges» qu'Appelfeld préfère taire, tant ils renferment l'horreur.

14 Passé maître dans l'art de la concision et de l'euphémisme, Appelfeld a développé dans son œuvre la dualité entre le dit et le non-dit, menant à son paroxysme le paradoxe qui consiste à prendre le silence comme objet de l'artéfact verbal qu'est le roman. C'est cette même dualité à laquelle renvoie Masha Itzhaki dans sa monographie Aharon Appelfeld: le réel et l'imaginaire ${ }^{10}$. Qu'est-ce que le «réel» et qu'est-ce que l'«imaginaire » dans une œuvre qui n'a cesse d'examiner les limites entre le récit factuel et le récit de fiction? Pour le dire avec Appelfeld :

Je viens d'un monde où le réel est précis et répétitif: les camps et la forêt. Mon monde réel dépassait de loin la puissance de l'imaginaire et, en tant qu'artiste, je n'ai pas eu besoin de stimuler mon imagination, mais au contraire, de la freiner, et à chaque fois que j'y parvenais, le résultat me semblait encore impossible, parce que tout était tellement incroyable que je me faisais l'impression d'être moi-même un être imaginaire [...] J'ai essayé à plusieurs reprises d'écrire "l'histoire de ma vie » dans les bois après mon évasion. Mais tous mes efforts ont été vains. Je voulais être fidèle à la réalité et à ce qui s'est vraiment passé. La chronique à laquelle j'ai abouti n'était qu'une structure assez faible. Le résultat était plutôt maigre, un récit imaginaire peu convaincant ${ }^{11}$.

15 Cette dualité entre le référentiel et le non-référentiel, le réel et l'imaginaire prend dès lors toute sa dimension dans le roman appelfeldien comme le va-et-vient entre les éléments biographiques et factuels/historiques proprement dits (le réel) et leur élaboration fictionnelle (l'imaginaire). Une réalité dépasse l'autre, s'impose au point d'effacer les limites qui existent entre elles. Ainsi, Primo Levi, au retour d'Auschwitz, souffre d'un cauchemar récurrent. Dans son rêve, la réalité paisible du 
quotidien s'efface : « Je suis à nouveau dans le Camp et rien n'était vrai que le Camp. Le reste, la nature en fleur, le foyer, n'était qu'une brève vacance, une illusion des sens $»^{12}$. Il en est de même pour Robert Antelme : «À peine commencions-nous à raconter que nous suffoquions. À nous-mêmes, ce que nous avions à dire commençait alors à paraître inimaginable. Cette disproportion entre l'expérience que nous avions vécue et le récit qu'il était possible d'en faire ne fit que se confirmer par la suite $»^{13}$.

L'« indicible», on le voit, se traduit par certaines expressions récurrentes qui reviennent dans les propos des survivants : "moi-même un être imaginaire ", "récit imaginaire », « illusion des sens », « inimaginable », « la réalité dépasse l'imagination ». C'est donc l'effacement des limites entre deux réalités, chacune aussi factuelle que l'autre, que suggère le « réel non-référentiel » des récits de l'indicible. Mais lequel des deux vécus est-il «réel» et lequel « imaginaire»? Est-ce la réalité des camps ou la réalité du quotidien dit « normal»?

\section{De la figure du Double à l'hésitation fantastique}

17 Les propos d'Appelfeld cités plus haut sont ceux qu'il a tenus dans un entretien publié dans le New York Times Book Review ${ }^{14}$ avec Philip Roth en 1988. Dans son roman Opération Shylock ${ }^{15}$, Roth met en récit un certain Aharon Appelfeld, écrivain israélien de son état, ami du narrateur, dénommé lui-même Philip Roth. L'Appelfeld fictionnel de Roth, n'a cesse de répéter les propos du véritable Appelfeld dans son interview pour le New York Times Review of Books, propos qu'Appelfeld l'écrivain se réapproprie quelques années plus tard dans Histoire d'une vie ${ }^{16}$, son récit autobiographique. À l'inverse du Temps des prodiges, Opération Shylock de Philip Roth est une fiction qui refuse de se définir comme telle. Double tour de passe-passe littéraire entre Roth et Appelfeld, il montre bien que les limites poreuses entre le fictionnel et le factuel vont bien au-delà des questions théoriques dont ils font l'objet. Si Roth, exubérant et provocateur, et Appelfeld, introverti et laconique, nous semblent de prime abord l'antithèse l'un de l'autre, la logorrhée de l'un le disputant au mutisme de l'autre, le trop-dit de l'Américain au nondit de l'Israélien, ils personnifient dans le même temps la figure du Double, cher à la littérature fantastique.

Grand thème d'Opération Shylock, les doubles prolifèrent dans ce roman jusqu'au vertige. Ainsi, le Philip Roth fictionnel, narrateur d'Opération Shylock, arrive à Jérusalem en janvier 1988 pour interviewer Appelfeld au moment même où deux événements majeurs tiennent en haleine la presse internationale réunie au grand complet à Jérusalem : d'une part la première Intifada, premier soulèvement palestinien contre l'occupation israélienne qui débute en novembre 1987, de l'autre, le procès de John Demjanjuk, dit "Ivan le Terrible», accusé de crimes contre l'humanité en tant qu'ancien gardien-tortionnaire du camp d'extermination de Treblinka. Ces deux événements, qui sont intégrés dans la trame de l'intrigue, lui confèrent une dimension historique sur laquelle Roth insiste par ailleurs dans sa préface au livre, signée P. R., où il indique non seulement que « ce livre est le compte rendu aussi fidèle que possible des événements que j'ai effectivement vécus entre ma cinquantième et ma soixantième année ${ }^{17}$, mais il va jusqu'à se réclamer de leur " vérité intrinsèque ». Jusqu'ici, rien de surprenant lorsqu'il s'agit d'une œuvre qui porte le sous-titre de Confession, dont le narrateur est identique à l'auteur dont la photo apparaît d'ailleurs sur la couverture en 
guise d'illustration. Mais Philip Roth est-il un narrateur « digne de confiance »? Cette "vérité intrinsèque » en est-elle une?

Les premières brèches de cette "vérité intrinsèque " apparaissent avec l'irruption d'un certain Philip Roth bis dans la vie de Philip Roth le narrateur. Ancien détective de Chicago, ce Double profite de son étonnante ressemblance physique avec le célèbre écrivain-narrateur pour s'en approprier le nom et en usurper l'identité. Réplique exacte de Roth, son Double marche comme lui, s'habille comme lui, parle comme lui et publie dans la presse des articles signés Philip Roth. Pour s'en démarquer, Roth I le narrateur désigne son Doppelgänger, Roth II, par le nom yiddish de Moishe Pipik. Il n'en demeure pas moins que Roth I s'interroge pour savoir si Roth II existe vraiment ou s'il s'agit d'une hallucination due à la consommation d'un somnifère vendu sous le nom d'Halcion ou d'un délire paranoïaque, effet secondaire ou retour d'une dépression nerveuse longuement décrite dans le premier chapitre ${ }^{18}$.

L'irruption soudaine de Philip Roth bis, figure du Double dans la vie du «vrai » Philip Roth nous permet-elle de définir Opération Shylock comme un récit fantastique ? Et si c'était le cas, le bon Dr. Jekyll qui se transforme en un hideux Mr. Hyde comme dans le roman de R.L.Stevenson et qui réapparaît ici dans une version juive américaine entretient-il une quelconque relation avec Appelfeld? Si nous prenons le dédoublement de Roth et d'Appelfeld en personnages réels et imaginaires dans ce roman comme indice supplémentaire, il n'est pas abusif d'établir une relation entre la figure du Double fantastique chez Roth, l'indicible comme « réel non-référentiel » et Le Temps des prodiges d'Appelfeld.

En effet, pour le dire avec Masha Itzhaki,

aucun roman d'Appelfeld ne peut être considéré comme autobiographique dans le sens classique du terme [...] Même dans son ouvrage le plus autobiographique, Histoire d'une vie, Appelfeld avertit son lecteur tout au début en disant: "Que le lecteur n'aille pas chercher dans ces pages une histoire de vie précise et structurée $»^{19}$.

Cette déclaration d'intention d'Appelfeld s'oppose donc clairement aux déclarations de Roth qui, on l'a vu, revendique la "vérité intrinsèque » de son récit, à la façon des auteurs réalistes du XVIII ${ }^{e}$ et du XIXe siècle. Mais Roth, qui a sans doute lu les maitres du réalisme n'en reste pas là. Pour mieux brouiller les pistes, dans la "Note au lecteur " qui clôture Opération Shylock dont le sous-titre, on le rappelle, est Confession, l'auteur prétend, contrairement à ses affirmations précédentes que «ce livre est une œuvre de fiction » et que « cette confession est un faux $»^{20}$.

Pour ajouter à la confusion, cette « Note au lecteur » qui, contrairement à la préface, n'est pas signée, précise toutefois que les entretiens avec Appelfeld ont effectivement été publiés dans le New York Times du 11 mars 1998. Que faut-il en conclure? Roth ne cesse de renverser les conventions canoniques du genre fantastique en se présentant non plus seulement comme son double fictionnel comme dans le cas d'une autofiction, mais en se plaçant comme son propre critique littéraire :

Je me retrouvais bientôt à me demander s'il valait mieux présenter le livre non comme une confession autobiographique qu'un certain nombre de lecteurs, à la fois hostiles et bienveillants, se sentiraient obligés de mettre en question pour des raisons de crédibilité, ou comme une histoire dont le but même était son improbable réalité, mais plutôt - prétendant que j'avais moi-même inventé ce qui m'avait été fourni à profusion, et gratuitement, par une réalité "super-inventive »- comme une fiction, une construction en forme de rêve éveillé dont l'auteur aurait 
déterminé le contenu latent de manière aussi délibérée que les aspects tout à fait évidents. Je pouvais même imaginer, en toute perfidie, présenter Opération Shylock comme un roman dans lequel une poignée de lecteurs astucieux verraient une chronique de l'hallucination déclenchée par l'Halcion, ce que même moi, un moment, au cours d'un des épisodes les plus étonnants de Jérusalem, j'étais presque arrivé à croire ${ }^{21}$. métaphorique, voire allégoric « fantastique » peut se traduire par « anormal », ce qui dépasse l'entendement, c'est la grande question existentielle posée par Roth: "Qu'est-ce qu'être 'normal' pour un juif?", qui nous permet d'interroger les conventions du genre fantastique en rapport avec l'oxymore des « prodiges » appelfeldiens.

\section{Le fantastique et la littérature du désastre}

Roger Caillois définit le fantastique comme «la rupture de l'ordre reconnu, irruption de l'inadmissible au sein de l'inaltérable légalité quotidienne ${ }^{26}$. Or, à y regarder de près, cette définition du fantastique n'est pas sans rappeler la coupure brutale entre le monde dit "normal» et celui de l'univers concentrationnaire que l'on trouve très souvent dans les témoignages des survivants de la Shoah. Est-il abusif, dès lors, de le décrire, en réutilisant les termes qu'applique Caillois au fantastique, comme "un scandale, une déchirure, une irruption insolite, presque insupportable dans le monde réel »? Autrement dit, il s'agit de transformer l'altérité radicale du fantastique en métaphore pour décrire la monstruosité bien réelle des camps. 
29 Notion qui se rapproche de ce que Sartre a désigné comme la « littérature de situations extrêmes $»^{27}$, la "littérature du désastre ", serait-elle un genre que l'on pourrait rattacher au fantastique comme une forme de rupture cognitive et à condition de faire une distinction très claire entre le fantastique de divertissement et le fantastique comme une forme du tragique?

Tzvetan Todorov le dit clairement dans son Introduction à la littérature fantastique :

Dans un monde qui est bien le nôtre, celui que nous connaissons, sans diables, sylphides, ni vampires, se produit un événement qui ne peut s'expliquer par les lois de ce même monde familier. Celui qui perçoit l'événement doit opter pour l'une des deux solutions possibles : ou bien il s'agit d'une illusion des sens, d'un produit de l'imagination et les lois du monde restent alors ce qu'elles sont; ou bien l'événement a véritablement eu lieu, il est partie intégrante de la réalité, mais alors cette réalité est régie par des lois inconnues de nous [...]. Le fantastique, c'est l'hésitation éprouvée par un être qui ne connaît que les lois naturelles face à un événement en apparence surnaturel ${ }^{28}$.

31 Mais Todorov nous met en garde : l'événement n'est donc surnaturel qu'en apparence, car «si ce que nous lisons décrit un événement surnaturel, et qu'il faille pourtant prendre les mots non dans le sens littéral, mais dans un autre sens qui ne renvoie à rien de surnaturel, il n'y a plus lieu pour le fantastique ${ }^{29}$.

En ce sens, si l'événement « surnaturel » est la Shoah, il est « surnaturel » au regard de son improbabilité, de son horreur, de son indicibilité, mais pas du point de vue de sa factualité. Todorov parle encore de l'« hésitation fantastique ». Cette " hésitation » est "commune au lecteur et au personnage qui doivent décider si ce qu'ils perçoivent relève ou non de la "réalité", telle qu'elle existe pour l'opinion commune ${ }^{30}$. Appelfeld le reconnaît d'ailleurs dans son interview avec Philip Roth: "On peut falsifier facilement les choses les plus vraies.» « Mais », et c'est ce grand, MAIS qui suit cette affirmation :

Comme vous le savez, la réalité dépasse toujours l'imagination de l'homme. Qui plus est, la réalité peut se permettre d'être incroyable, inexplicable, exagérée. Mais l'œuvre de l'imagination ne peut, à mon grand regret, se permettre le même genre de choses. La réalité de la Shoa a dépassé tout ce que l'on peut imaginer. Si je rapportais fidèlement les faits, personne ne me croirait ${ }^{31}$.

L'« hésitation fantastique » n'est donc pas du côté du témoin qui, lui, ne doute pas de la réalité de son vécu, mais du côté de son interlocuteur qui met en doute la véracité du témoignage. On comprend mieux, dès lors, les propos de Primo Levi et Robert Antelme cités plus haut. C'est le refus d'écouter les témoins, de les croire, qui devient une nouvelle source de souffrance et d' « hésitation fantastique ». Si dans un premier temps cette "hésitation" est bien celle du témoin qui subit l'irruption de ce qui est inadmissible et invraisemblable, elle prend une nouvelle forme, presque aussi terrible, lorsque cette situation prend fin. Cette fois, pour les survivants, l'« hésitation» fantastique ne naît plus de l'irruption de l'invraisemblable ou du surnaturel dans le monde dit normal, mais au contraire, de l'irruption du monde «normal » du quotidien dans le monde invraisemblable des camps. Autrement dit, ce qui ressort des propos des survivants c'est qu'après le désastre, c'est le monde du quotidien et de l'opinion commune qui devient invraisemblable à leurs yeux. Le monde des camps et de la persécution devient le seul monde possible et vraisemblable.

34 Il ne nous semble donc pas abusif de créer un rapprochement entre la catégorie cognitive de l'«hésitation fantastique » et la «littérature du désastre », catégorie 
thématique et taxinomique dans laquelle Masha Itzhaki propose de classer l'œuvre d'Appelfeld, car celle-ci, écrit-elle, « n'obéit plus, ne peut pas obéir, à la classification littéraire conventionnelle qui insistait sur la distinction entre une écriture à caractère documentaire - dont l'objectif est d'apporter un témoignage personnel et donc de constituer un chapitre autobiographique - et un récit qui porte sur la fiction selon les règles de la fiction $»^{32}$.

Or, nous l'avons dit, c'est précisément le non-dit, la page blanche qui sépare les deux récits qui forment Le Temps des prodiges qui symbolise l'« indicible». Indicible, car la réalité dépasse l'imaginaire. Mais le paradoxe appelfeldien consiste, précisément, à raconter et non pas à se taire. Dans un entretien publié en 1979 à la suite de la publication du Temps des prodiges, Appelfeld reconnaît ne jamais avoir décrit les choses comme elles s'étaient passées :

Toutes ces années j'ai essayé de reconstituer mon enfance, de la restituer fragment par fragment, de remplir les espaces blancs. Le Temps des prodiges est une tentative de reconstruire l'enfance. L'œuvre de restitution est nécessaire puisque la certitude est réduite et l'obscurité est grande. C'est un travail d'archéologie que j'ai effectué en moi-même. En réalité, c'est un processus créatifi33.

\section{Regarder le soleil en face}

Sous cet aspect, Appelfeld se distingue donc nettement de Primo Levi, Robert Antelme, Jorge Semprun ou encore Élie Wiesel, plus âgés que lui, dont les souvenirs étaient plus précis et dont les témoignages revendiquent le statut de récit factuel ${ }^{34}$. Les années de déportation et de fuite constituent un trou noir dans la mémoire d'Appelfeld, d'où la dénotation nulle, d'où le réel non-référentiel de l'indicible.

Une fois de plus, l'hésitation "fantastique » de l'indicible efface ainsi les oppositions vérité-mensonge, réel-irréel, vraisemblable-invraisemblable, toutes aussi présentes dans l'œuvre d'Appelfeld que dans celle de Roth. C'est donc toujours cette même page blanche du Temps des Prodiges qui renvoie subrepticement à la question que se pose le Philip Roth narrateur dans Opération Shylock sur Appelfeld :

Ses histoires sont-elles exactes, sont-elles vraies? Moi, je ne pose jamais de questions sur leur véracité. Je crois plutôt que c'est du roman et, comme c'est souvent le cas, le roman fournit à celui qui l'invente un mensonge par lequel il exprime son indicible véritée ${ }^{35}$.

En effet, l'indicible, selon Alexis Nouss, n'est pas une catégorie qui pose l'impuissance du langage face à l'horreur. Au contraire, « elle devient le motif profond, la motivation positive qui permet le récit, qui oblige et suscite le récit. Le récit de l'indicible n'opère pas négativement : il raconte l'indicible, sert à le montrer. $\|^{36}$ Mais montrer n'est pas représenter. D'où la conclusion de Nouss citée plus haut: «L'indicible mine conceptuellement l'idée de la représentation [...] l'indicible n'est pas représentable ou irreprésentable, il suggère un réel non référentiel.» C'est par une image saisissante qu'il cite d'Aharon Appelfeld que Nouss illustre cet «indicible »: "Cette mort-là [...] est semblable au soleil, on ne peut en regarder la lumière en face. $\|^{37}$

Ainsi, la page blanche qui sépare les deux parties du Temps des prodiges d'Appelfeld constitue l'illustration de "l'invisible qui se place sous le signe du visuel». La page blanche se voit, mais elle est vide, son texte est invisible. Mais ce qui est invisible pour le lecteur qui n'a pas subi lui-même l'expérience de la persécution est visible uniquement pour celui qui a regardé une fois le soleil en face. 


\section{BIBLIOGRAPHIE}

\section{Textes de fiction, témoignages et interviews}

ANTELME, Robert (1978), L'espèce humaine, Paris, Gallimard, coll. « Tel ».

APPELFELD, Aharon (1978), Tor ha-pla'ot, Tel Aviv, Hakibbutz Hameuchad (trad. de l'hébreu Arlette Pierrot, Le Temps des prodiges, Paris, Belfond, 1985, réimp. Paris, Seuil, coll. « Points » 2004).

APPELFELD, Aharon (1979), Massot be-guf rishon (« Essais à la première personne »), Jérusalem, Hasifryah Hatsionit.

APPELFELD, Aharon (1999), Histoire d'une vie, trad. de l'hébreu Valérie Zenatti, Paris, Gallimard, 2004.

APPELFELD, Aharon (1994), Beyond Despair, Three Lectures and a Conversation with Philip Roth, New York, Cromm International.

GOETHE, Johann Wolfang (1782), Der Erlkönig in Sämtliche Werke, Briefe, Tagebücher und Gespräche, Tome 2. Berlin, Deutscher Klassiker-Verlag, 1987, p. 107-108. Traduction française de la poésie in Michel Tournier (1970), Le roi des Aulnes, Paris, Gallimard, coll. « Folio », 2010, p. 497.

HILSENRATH, Edgar (2012), Nuit, trad. de l'allemand J. Stickan et S. Zilberfarb, Paris, Attila.

KAFKA, Franz (1958), Briefe 1902-1924 in Gesammelte Werke, vol. 9, Francfort-sur-le-Main, Fischer.

LEVI, Primo (1987), Si c'est un homme, trad. de l'italien M Schruoffeneger, Paris, Julliard.

LEVI, Primo (1966) , La Trêve, trad. de l'italien E. Genevois-Joly, Paris, Grasset.

Rотн, Philip (1995), Opération Shylock : Une Confession, trad. de l'américain L. Bitoun, Paris, Gallimard, coll. « Folio ».

Rотн, Philip (28.02.1988) "Walking the Way of the Survivor-A Talk with Aharon Appelfeld", The New York Times Review of Books. En français in Philip Roth (2006), Parlons travail, trad. Josée Kamoun, Paris, Gallimard, coll. « Folio ».

TOREN, Orly (05.05.1989), « Be-heqah shel Katerina » (« Au sein de Katerina - Entretien avec Aharon Appelfeld »), Yedioth Yerushalayim.

\section{Essais, théorie, critique}

BEN-MORDECHAI, Yitzhak et PARUSH, Iris, (1997) Beyn kfor le-'ashan («Entre givre et fumée. Études sur l'œuvre d'Aharon Appelfeld »), Beer Sheva, Université Ben Gourion.

BLEIKASTEN, André (2001), Philip Roth - Les ruses de la fiction, Paris, Belin.

CAILLOIS, Roger (1966), « De la féerie à la science-fiction » in R. Caillois (dir.), Anthologie du fantastique, T. I, Paris, Gallimard.

DOUBROVSKY, Serge (25.04.2003) « Pourquoi l'autofiction? », Le Monde des livres.

ITZHAKI, Masha (2011), Aharon Appelfeld : le réel et l'imaginaire, Paris, L'Harmattan.

Nouss, Alexis (1998), « Les récits de l'indicible » in Récit et connaissance, Lyon \& Montréal, Presses Universitaires de Lyon \& Méridien.

SARTRE, Jean-Paul, (2003), « Situation de l'écrivain en 1947 », in Qu'est-ce que la littérature ? Paris, Gallimard, coll. « Folio-Essais », 2003, p. 169-295. 
SCHWARTZ, Yigal (1996), Qinat ha-yahiid we-netsaḥ ha-ševeț ( Élégie de l'individu et éternité de la tribu - La vision du monde d'Aharon Appelfeld »), Jérusalem, Keter et Magness.

TODOROV, Tzvetan (1970), Introduction à la littérature fantastique, Paris, Seuil, coll. « Poétique ».

\section{NOTES}

1. Orly Toren, «Au sein de Katerina - Entretien avec Aharon Appelfeld», Yedioth Yerushalayim, 5 mai 1989 (en hébreu).

2. Aharon Appelfeld, Katerina, Jérusalem, Keter, 1989. Trad. française de Sylvie Cohen, Paris, Gallimard, 1996.

3. Aharon Appelfeld, Tor ha-pla'ot, Tel Aviv, Hakibbutz Hameuchad, 1978. Trad. française Arlette Pierrot, Le Temps des prodiges, Paris, Belfond, 1981.

4. Préface de Marianne Véron in Aharon Appelfeld, Le Temps des prodiges, op. cit. p. 7.

5. Alexis Nouss, "Les récits de l'indicible» in Récit et connaissance, Lyon \& Montréal, Presses Universitaires de Lyon/Méridien, 1998, p. 204-205.

6. Art. « prodige ", Nouveau Petit Robert, 1995.

7. Préface de l'auteur in Primo Levi (1947), Si c'est un homme, trad. de l'italien Martine Schruoffeneger, Paris, Julliard, 1987, p. 7.

8. Franz Kafka, Briefe 1902-1924 in Gesammelte Werke, vol. 9, Francfort-sur-le-Main, Fischer, 1958, p. 337. Traduction citée: «Lettre à Max Brod », juin 1921, in Franz Kafka, Oeuvres complètes, trad. de l'allemand J. P. Danès, C. David, M. Robert, A. Vialatte, Paris, «La Pléiade ", T. 3, 1984, p. 1087.

9. J.W. Goethe (1782), »Der Erlkönig«, in Sämtliche Werke, Briefe, Tagebücher und Gespräche, Tome 2. Berlin, Deutscher Klassiker-Verlag, 1987, p. 107-108. Traduction française de la poésie in Michel Tournier (1970), Le roi des Aulnes, Paris, Gallimard, coll. « Folio », 2010, p. 497.

10. Masha Itzhaki, Aharon Appelfeld : le réel et l'imaginaire, Paris, L'Harmattan, 2011.

11. Philip Roth, "Walking the Way of the Survivor: A Talk with Aharon Appelfeld", The New York Times Review of Books, 28 février 1988. En français in Philip Roth, Parlons travail, trad. Josée Kamoun, Paris, Gallimard, coll. « Folio », 2006.

12. Primo Levi (1963), La Trêve, trad. de l'italien Emmanuelle Genevois-Joly, Paris, Grasset, 1966, p. 245.

13. Robert Antelme, L'espèce humaine, Paris, Gallimard, coll. « Tel », 1978, p. 9.

14. Voir note 11.

15. Philip Roth (1993), Opération Shylock: Une confession, trad. de l'américain Lazare Bitoun, Paris, Gallimard coll. « Folio », 1997.

16. Aharon Appelfeld, Histoire d'une vie, trad. de l'hébreu Valérie Zenatti, Paris, Éditions de L'Olivier, 2004.

17. Philip Roth, Opération Shylock, op. cit. p. 13.

18. Ibid., p. 9. L'effondrement psychique réel de Roth l'écrivain est d'ailleurs attesté par le livre de sa compagne "référentielle» de l'époque, l'actrice anglaise Claire Bloom, protagoniste du roman et objet de la dédicace d'Opération Shylock .

19. M. Itzhaki, Aharon Appelfeld : le réel et l'imaginaire, op. cit. p. 17.

20. Philip Roth, Opération Shylock, op. cit., « Note au lecteur », p. 653.

21. Ibid., p. 587. C'est Philip Roth qui souligne.

22. André Bleikasten, Philip Roth: les ruses de la fiction, Paris, Belin, coll. «Voix américaines ", 2001, p. 93.

23. Philip Roth, Opération Shylock, op. cit. p. 43-46.

24. Jean-Paul Sartre, Réflexions sur la question juive, Paris, Gallimard, 1954.

25. Serge Doubrovsky, « Pourquoi l'autofiction?», Le Monde des Livres, 25 avril 2003. 
26. Roger Caillois, « De la féerie à la science-fiction » in: R. Caillois (dir.), Anthologie du fantastique, T. I, Paris, Gallimard, 1966, p.8.

27. Jean-Paul Sartre (1948), « Situation de l'écrivain en 1947 », in Qu'est-ce que la littérature ?, Paris, Gallimard, coll. « Folio-Essais », 2003, p. 169-295.

28. Tzvetan Todorov, Introduction à la littérature fantastique, Paris, Seuil, coll. « Poétique », 1976, p. 29. Nous soulignons.

29. Philip Roth, Opération Shylock, op. cit. p. 69.

30. Tzvetan Todorov, Introduction à la littérature fantastique, op. cit. p. 46.

31. Philip Roth, "Walking the Way of the Survivor: A Talk with Aharon Appelfeld", art. cit.

32. M. Itzhaki, Aharon Appelfeld: le réel et l'imaginaire, op.cit. p. 28.

33. Cité par Yigal Schwartz in Élégie de l'individu et éternité de la tribu, Jérusalem, 1996, p. 37 (en hébreu).

34. Signalons la publication récente (et tardive) en français de l'excellent roman Nuit (1964) d'Edgar Hilsenrath, trad. de l'allemand Jörg Stickan et Masha Zilberfarb, Paris, Attila, 2012, qui décrit la Transnistrie dans un style cru et très réaliste.

35. Philip Roth, Opération Shylock, op. cit. p. 87.

36. Alexis Nouss, «Les récits de l'indicible», op.cit. p. 207.

37. Ibid.

\section{RÉSUMÉS}

C'est la marge étroite entre « le réel et l'imaginaire » selon le titre de l'essai de Masha Itzhaki sur Appelfeld que nous souhaiterions explorer à partir de la relation entre Le Temps des prodiges d'Appelfeld et Opération Shylock de Philip Roth. Si la vrai/fausse "confession" de Roth se présente comme le détournement vertigineux des codes du fantastique, de l'autobiographie et de l'autofiction, le roman d'Appelfeld pousse les limites du réalisme par l'interrogation que suscite son titre énigmatique qui renvoie au surnaturel. Cuuvre qui évoque la Shoah sans la représenter, le roman d'Appelfeld correspond à ce que Sartre a défini comme une « littérature des situations extrêmes ». Néanmoins, par son titre et la page blanche qui sépare ses deux parties et renvoie à l'« indicible » de la Shoah, ne sollicite-t-elle pas aussi le fantastique qui, selon Todorov, n'est pas nécessairement une configuration imaginaire, mais plutôt « l'hésitation éprouvée par un être qui ne connaît que les lois naturelles, face à un événement en apparence surnaturel »? Philip Roth, critique littéraire tout autant qu'écrivain, n'a t-il pas bien cerné cette dualité en créant dans Opération Shylock un Appelfeld fictionnel, le «double » bienveillant de son homonyme réel aux côtés d'un Roth affublé d'un "double maléfique», indiquant ainsi qu'Appelfeld transcende l'événementiel qui lui a valu injustement le titre réducteur d'« auteur de la Shoah »?

In the present paper I wish to explore the narrow borderline between history and fiction in Aharon Appelfeld's The Age of Wonders (1978) and Philip Roth's Operation Shylock (1988). Could Appelfeld's Age of Wonders, a seemingly realistic novel about the Holocaust, be at once what Sartre called "a literature of extreme situations" and belong to the fantastic genre? If we accept Todorov's definition of the latter as "an hesitation experienced by a person who knows only the laws of nature, confronting an apparently supernatural event", it is precisely the absence of any supernatural events, emphasized by the empty page separating Appelfeld's protagonist's childhood and adulthood that raises the question weather the Holocaust, never explicitly 
represented in the novel, could be interpreted as the apocalyptic "age of wonders".

In contrast, Roth's musings about Jewish history in the 20th century in his Operation Shylock are structured as a re-appropriation and a parody of the classical fantastic genre, where Appelfeld, as well as Roth himself, are set as fictional characters and doppelgängers of themselves. In both works, the authors show the fallacy of realism to represent history, and in transcending the socalled "factual" realm, blur the boundaries between history and fiction, autobiography and autofiction.

אמר זה עוסק בשאלת הגבול בין "מציאות לבדיון" ביצירת אפלפלד באמצעות השוואה בין יצירתומ

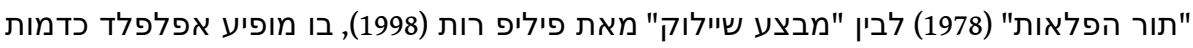

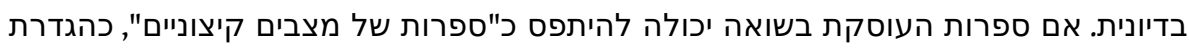

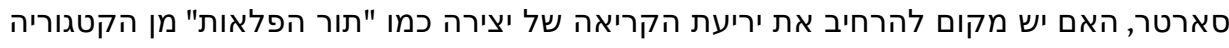

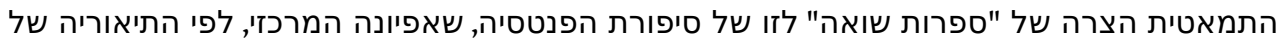

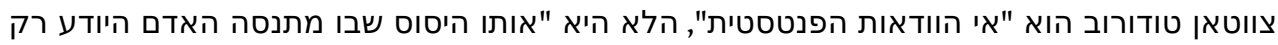

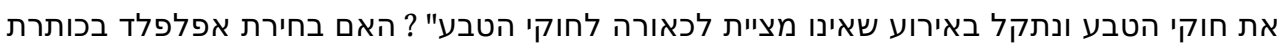

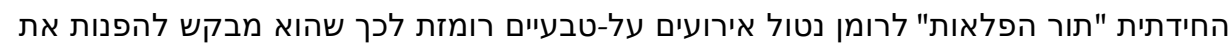

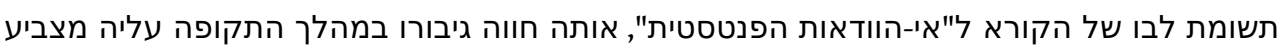

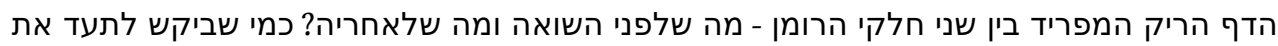

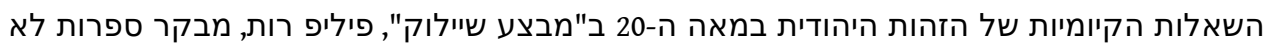

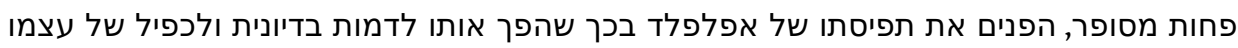

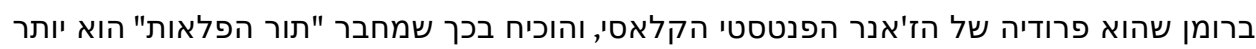

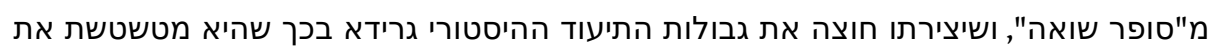

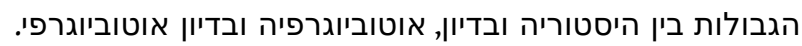

\section{INDEX}

Thèmes : littérature

Index géographique : Transnistrie

Keywords : Appelfeld Aharon (1932-), Roth Philip (1933-), fantastic literature, “Doppelgänger”, Holocaust, Transnistria, autobiography, autobiographical fiction

Mots-clés : Appelfeld Aharon (1932-), Roth Philip (1933-), le fantastique, le double, autobiographie, autofiction

\section{מילות מפתח}

אהרון אפלפלד, פיליפ רות, סיפורת פנטסטית, שואה, טרנסניסטריה, אוטוביוגרפיה:

Index chronologique : Shoah 